\title{
CRISNEJA, UN MERIDIONALISMO EN EL ESPAÑOL DE AMÉRICA
}

\author{
JuAN ANTONio Frago Gracia
}

Si se consulta el DRAE en su última edición, aparecerá en primer lugar una entrada crisneja que sólo remite a la de crizneja 'trenza de cabellos', 'soga o pleita' ${ }^{1}$. Aunque se eche en falta alguna nota, de arcaísmo o de regionalismo - en cambio se atribuye el carácter de anticuado al adj. encrisnejado 'dícese del cabello u otra cosa que está hecha trenzas'-, la mera variación gráfica revela que no se trata de un término de difusión general en la actualidad, sino que, todo lo más, el referido doblete habrá estado adscrito a un dominio seseoso o ceceoso, tal vez de ambos fenómenos, y que quien introdujo y mantuvo estas formas en el diccionario académico no se encontraba muy familiarizado con ellas, pues prefirió poner las definiciones bajo la variante grafémicamente ceceosa. Terreros no recoge crisne$j a$, pero sí su derivado encrisnejado 'hecho trenza', indicio de que probablemente la voz simple también existía en el español de finales del siglo XVIII, aunque, casi con toda seguridad asimismo, no fuera de uso común ${ }^{2}$. Y, por referencia al primer tercio de dicha centuria, el Autoridades,

\footnotetext{
${ }^{1}$ Real Academia Española, Diccionario de la lengua española, Madrid, Espasa-Calpe, 1992, 21. a edición, s. vv. También se halla una entrada crezneja, con simple remisión a la de crizneja. Por el predominio documental de la variante con $-s$ y por la preferencia normativa a favor del seseo, está claro que el registro lexicográfico debe corresponderle a crisneja, teniendo en cuenta además lo que sugiere el castellano-manchego grisneja abajo aducido. A no ser que se crea, en contra de todos los datos aquí reunidos, que se trata de un término estándar.

${ }^{2}$ Esteban de Terreros y Pando, Diccionario castellano con las voces de ciencias y artes (1786-1793), edición facsímil, Madrid, Arco Libros, 1987, t. II, pág. 36. Terreros ni siquiera tiene seguridad en la consideración semántica de encrisnejado, pues añade la extraña advertencia de que «otros toman el cast. por lo mismo que el que tiene varios o diversos cabellos». Es probable que este lexicógrafo manejara la definición del Autoridades, pero también parece moverse con menor precisión en todo lo tocante a dicho caso léxico que el primer redactor académico.
} 
si bien tampoco da cabida en artículo independiente al vocablo del que me ocupo, lo incluye en el de encrisnejado 'hecho trenza con los cabellos u otra cosa', cuando explica: «es formado de la preposición en y del nombre cris$n e j a$, que en Indias significa la trenza» ${ }^{3}$. Queda así claramente identificada en fuente lexicográfica española del setecientos la palabra crisneja, cuya implantación se atribuye al mundo indiano con el referido significado de 'trenza', como la de encrisnejado, que viene autorizado en cita del P. Acosta ${ }^{4}$.

El DCECH reúne importante información histórica y dialectológica acerca de la voz en cuestión, señalando que el diccionario de la Academia admitió en 1783 crezneja 'pleita pequeña hecha de esparto cocido y majado' y crizneja 'soga o trenza hecha de mimbres', con la advertencia de que crisneja, en simple remisión a la variante con $-z$, demoró su recepción hasta la edición de $1884^{5}$. En cuanto a su documentación, la saca del vocabulario de Pedro de Alcalá («crisneja de tres cuerdas», en 1505), así como de los literatos andaluces Lope de Rueda y Góngora, constantemente en la variante con $-s^{6}$, concluyendo Corominas que «hoy, como en el Siglo de

\footnotetext{
${ }^{3}$ Real Academia Española, Diccionario de Autoridades (1726-1739), edición facsímil, Madrid, Editorial Gredos, 1969, t. II, pág. 452.

${ }^{4}$ Con el pasaje «venían a criarse en el cabello unas trenzas, que parecían clines de caballo encrisnejadas"), referido a las costumbres de los sacerdotes mexicas, el mismo que para su documentación emplea el $D E C H$, donde se dice que crisneja falta en el Autoridades: J. Corominas, con la colaboración de José A. Pascual, Diccionario crítico etimológico castellano e hispánico, Madrid, Editorial Gredos, 1980-1991, s. v. crizneja.

5 Las preferencias académicas por crizneja sin duda han determinado su elección en el $D E C H$ como lema del correspondiente artículo léxico. La etimología de este término dista mucho de ser definitiva en las hipótesis que Corominas formula, todas ellas interesantes, desde luego. El germanesco crisna no tiene por qué ser necesariamente un regresivo de crisne$j a$, en vista de un canario clina 'clin (crin)' puesto en relación con el portugués por Cristóbal Corrales Zumbado, Dolores Corbella Díaz y M. ${ }^{a}$ Ángeles Álvarez Martínez, Diccionario diferencial del español de Canarias [DDEC], Madrid, Arco Libros, 1996, pág. 383. Muy bien puede ser crisneja una simple sufijación de crin, hecha quizá por influjo de cerneja, más todavía teniendo en cuenta el medieval crisne mencionado en la nota 13; si no es esa misma palabra con metátesis creneja, que Corominas atestigua el año 1546, precisamente en autor andaluz. Su $-s$, si no se debe a cruce analógico con otro vocablo, constituiría inicialmente un caso de ultracorrección, explicable en hablas meridionales que tienden a la pérdida de esta implosiva, dicho sea esto también a título de mera hipótesis. Por lo que a su formación se refiere, es evidente que la variación crisneja-crizneja nada tiene que ver con las del tipo biscocho-bizcocho, bisnieto-biznieto, o cualesquiera otras que contaran con una sibilante implosiva desde su étimo. Y, claro está, a un hablante confundidor en su pronunciación nada le importa que la palabra se escriba con $-s$ o con $-z$.

${ }^{6} \mathrm{La}$ impronta meridional sobre el vocabulario de Pedro de Alcalá es bien conocida. Como él, pondrá crisneja Lope de Rueda con el sentido de 'crines', igual que Góngora en cuanto a la forma, pero con significado, aunque figurado («crisneja de tales tres nietas»), similar al registrado en Alcalá. Y está el crenejas del andaluz Chacón, que Corominas relaciona con cerneja y crizneja. En cuanto a la existencia de esta última variante en Minsheu (1623) y en la edición académica de 1783, nada sabemos sobre las fuentes en que ambos registros lexicográficos se fundamentan.
} 
Oro, el vocablo se emplea en Andalucía (crineja 'soga de cinco liñuelos') $\mathrm{y}$ en varios puntos de América: clineja «trenza» en el Cibao dominicano, clineja 'la trenza o crencha del cabello en las mujeres, tejida en tres ramales o madejas gruesas' en Venezuela, y crisneja se emplea en el Oeste argentino, en San Juan y en Mendoza» ${ }^{7}$.

La misma extensión americana de crisneja que estas notas sugieren, así como su diversificación semántica, casi irremediablemente remiten a un asentamiento léxico verificado en los primeros decenios del período colonial, en el muy probable supuesto de que se esté ante un americanismo originado en un regionalismo español, y no será difícil ampliar la geografia lingüística de crisneja en América, en vista de que Morínigo localiza en Puerto Rico clineja 'crizneja o soga en forma de trenza que se hace del cogollo de la palma para hamacas y otros usos' ${ }^{8}$. No sólo eso: los autores del TLEC testimonian como canarismo las variantes clineja 'crines' y crineja 'crines del cuello y de la cola del caballo' 9; y en su DDEC bajo la voz clineja observan que en Argentina se halla con el significado de 'pelo de la cola de los caballos', como 'mechón de pelos' en la República Dominicana, como 'trenza de cabellos, crencha' en Cuba y Venezuela, mientras que para crineja apuntan la acepción 'pedazo de hilo que se desprende de una tela' junto a la tinerfeña de 'mechón de pelo', añadiendo el sentido 'trenza de cabellos, crencha al uso boliviano' ${ }^{10}$.

Si fijamos nuestra atención en la documentación indiana, pronto se com-

\footnotetext{
${ }^{7}$ Para el uso argentino Corominas refiere el significado de la descripción dieciochesca de un jesuita: «Se hace de la totora ya madura... una especie de cuerda trenzada de tres hilos y cada hilo compuesto de muchas hojas o ramas de esta planta... Esta cuerda... viene destinada a servir de cubierta o de forro a los barriles de greda... en que se conducen los vinos y aguardientes... esta crisneja, que es en el país su nombre, es un reparo y preservativo con el que se preservan los caldos... de... la fuerza del calor en viajes tan dilatados». Aunque en el $D C E C H$ sólo se aporta el actual crineja, tomado de Alcalá Venceslada, este autor en su obra también registra las variantes quinea 'juego de naipes parecido al rentoy y en el que intervienen cinco jugadores' (provincia de Huelva), quineja 'trenza de pelo' y quinneja 'crineja' (provincia de Jaén), quinnejuela 'crineja de ramalillos más delgados', quirneja-quinneja 'soga de cinco manojos': Vocabulario andaluz, Madrid, Editorial Gredos, 1980, reimpresión, pág. 517. En cuanto a Venezuela, todavía están muy vivas las formas crineja y clineja, ésta mucho más difundida: 'cabello peinado en forma de trenza' (general), 'trenza hecha con fibras secas para elaborar trabajos de cestería', sacar las clinejas 'golpear a una mujer' (Oriente): Rocío Núñez y Francisco Javier Pérez, Diccionario del habla actual de Venezuela, Caracas, Universidad Católica Andrés Bello, 1994, págs. 126, 146.

${ }^{8}$ Marcos A. Morínigo, Diccionario de americanismos, Barcelona, Muchnik Editores, 1985, pág. 138.

${ }^{9}$ Cristóbal Corrales Zumbado, Dolores Corbella Díaz y M. ${ }^{\mathrm{a}}$ Ángeles Álvarez Martínez, Tesoro lexicográfico del español de Canarias, Madrid-Canarias, Real Academia Española-Gobierno de Canarias, 1996, 2. ${ }^{\text {a }}$ edición, t. I, págs. 869, 959.

${ }^{10}$ DDEC, págs. 383, 424. También señalan esta variante crineja en la República Dominicana, Argentina, Cuba y Venezuela con las acepciones correspondientes a clineja.
} 
probará que la sincronía dialectal se sustenta en consistente fundamentación histórica. Efectivamente, un texto de Nueva Granada del año 1775 determina la existencia de esta palabra con significado figurado: «laboreada (la cinta) de oro o plata... y hecho todo una crisneja» ${ }^{11}$; y el periódico limeño Mercurio Peruano en 1794 menciona dicho término como hidrónimo de Cajamarca: «Riegan este partido muchos ríos. Los principales son el de Crisnejas, que está al Este, y, recibiendo otros muchos, desagua en el Marañón» ${ }^{12}$. Retrotrayéndonos al siglo fundacional del español americano, hallaremos la siguiente documentación costarricense de 1599: «3 enjalmas y lazos y sobrecarga y crisneja para aderezallos» ${ }^{13}$. Por otro lado, el P. Acosta no sólo emplea el encrisnejado referido por Corominas; también se muestra usuario de la forma sustantiva: «Éstas y otras mil maneras que tienen de pasar los ríos ponen cierto miedo cuando se miran, por parecer medios tan flacos y frágiles, pues en efecto son muy seguros. Puentes ellos no las usaban sino de crisnejas y paja» ${ }^{14}$. De 1603 a 1609 es el texto cronístico de Lizárraga, en el cual reiteradamente se emplea la variante crezneja, sin que por ello desconozca la de crizneja: «el primero [río] es el de Villcas, a 16 leguas de Guamanga; en tiempo de aguas poderoso, pásase por puente de creznejas; en tiempo de seca se vadea», "más adelante llegamos al río de Aporímac; éste también no se vadea; pásase por una puente de creznejas asaz larga y angosta», "es río grande y pocas veces se vadea, de gruesa agua; pásase por puente de criznejas, sin riesgo alguno», «y lo era de las puentes, entre las cuales, fuera de las creznejas, hay en ríos grandes, donde no se pueden hacer puentes, una manera de pasarlos jamás inventada, si no es en este reino del Perú» ${ }^{15}$. Por el término en $-s$, con el que se ve extraordinariamente familiarizado, se inclinaría el indio peruano Huaman Poma en la crónica que terminó de escribir el año 1615: «puente de crisnejas grandes que abía en tienpo del Ynga, como es de Bonbam, Xauxa, Ango Yaca...», «llebó yndios de deferentes castas y fue haziendo camino

\footnotetext{
11 Peter Boyd-Bowman, Léxico hispanomericano del siglo XVIII, Madison, 1982, pág. 804.

${ }^{12}$ La obra del obispo Martínez Compañón sobre Trujillo del Perú en el siglo XVIII, edición facsímil, Madrid, Ediciones Cultura Hispánica, Madrid, 1994, Apéndice II, núm. 335, del 20 de marzo de 1794, pág. 186.

${ }_{13}$ Peter Boyd-Bowman, Léxico hispanoamericano del siglo XVI, Madrid, 1972, pág. 239. Este hispanista pone crisueja donde sin el menor género de duda se leerá crisneja, con una deficiente transcripción de $n$ por $u$, nada rara por lo demás: en el mismo $D E C H$ se refiere la mala lectura de crisue por un medieval crisne "crin'.

${ }_{14}$ José de Acosta, Historia natural y moral de las Indias (Sevilla, 1590), edición de José Alcina Franch, Madrid, Historia 16, 1987, pág. 197.

${ }^{15}$ Reginaldo de Lizárraga, Descripción del Perú, Tucumán, Río de la Plata y Chile, edición de Ignacio Ballesteros, Madrid, Historia 16, 1987, págs. 169, 170, 180, 247. Se trata de los puentes o pasarelas colgados de dos gruesas sogas, hechas de la hoja seca de cierta palmera pequeña, que los indios peruanos tiran entre las orillas de los ríos.
} 
más adentro y llegó a un río grande y hizieron puente de crisnejas y pasaron a la otra uanda y llegó al valle llamado Uilcapampa», «mandó las puentes de cal y canto de la ciudad de Lima y de Xauxa y de Ango Yaco y de Amancay, que fueron todos de cantería, y otras puentes de crisnejas» ${ }^{16}$.

Ninguna duda cabe, pues, de que esta voz es de profunda raigambre americana, algo en cierto modo corroborado por su diferenciación semántica, ya verificada en las notas documentales arriba aducidas, así como por la derivación léxica a que dio lugar, desde antiguo comprobada igualmente. Los dos extremos de variación léxica, el semántico y el formal, se dan no sólo en la obra del P. Acosta, sino también en la de Lizárraga con la variante crizneja, para el dominico extremeño tanto 'especie de soga gruesa', según se ha visto, como 'trenza de cabello', significado que concuerda con el de criznejado 'trenzado', asimismo conocido de este autor: "salieron a ellos trece indios vestidos de blanco, manta y camiseta, con sus arcos y flechas; el cabello largo, criznejado, y en las criznejas flechas largas» ${ }^{17}$.

Polisemia y derivación léxica son fenómenos que, concurrentes en una misma palabra, aseguran su popularización, y, por cuanto a crisneja concierne, tal rasgo está de acuerdo igual con sus menciones documentales que con su expansión americana actual. Las fuentes indianas revelan las formas crezneja y crizneja, meras variantes por alteración en el timbre de una vocal átona, así como crisneja, con mucho la más frecuente y simple reflejo de una alternancia entre $-z$ y $-s$ propia de hablantes ceceosos y seseosos, que también se produce en los derivados criznejado, encrisnejado, los cuales a su vez exigen un previo verbo crisnejar, encrisnejar.

Del mediodía peninsular pudo pasar, pues, a Indias, crisneja, uno de tantos americanismos léxicos de procedencia regional que en el Nuevo Mundo perdieron su particularismo originario, adquiriendo muy pronto una gran extensión territorial y siendo rápidamente admitidos por todos los grupos de emigrantes, por los criollos y aun por los indios españolados: muchos de estos términos incluso se revistieron de otras referencias significativas, adaptados a la realidad americana ${ }^{18}$. Es igualmente el caso de crisneja, que desde Andalucía se llevaría también a Canarias, cuyas hablas influyeron con este vocablo en las americanas, tal vez de manera más acusada a partir del

\footnotetext{
${ }^{16}$ Felipe Huaman Poma de Ayala, Nueva crónica y buen gobierno, edición facsímil, París, Institut d'Ethnologie, 1968, reimpresión, págs. 357, 406, 439.

${ }_{17}$ Reginaldo de Lizárraga, Descripción del Perú, Tucumán, Río de la Plata y Chile, pág. 458. En esta crónica también se documentan las voces crencha, crin, maroma y soga con sus correspondientes significados básicos (págs. 73, 125, 192).

18 Peter Boyd-Bowman todavía añade dos documentaciones colombianas más recientes, una de 1867 que dice «tejía crezneja para sombreros», y otra de 1896 que reza «l'agarró por la criznejita y echó a jalar», como se ve con los significados de 'trenza vegetal' y 'trenza de cabello', respectivamente: Léxico hispanoamericano del siglo XIX, Madison, 1984, págs. 796, 802.
} 
siglo XVIII. Las concomitancias entre el modismo canario y el correspondiente uso léxico del área caribeña permiten aventurar esta hipótesis histórica.

En cuanto al andalucismo de este término, aparte de lo que el aparato documental en tal sentido sugiere, conviene señalar que en las definiciones académicas desde 1783 se incluye la voz pleita, según Alvar propia de Andalucía occidental y ya presente en los materiales nebrisenses como empleita ${ }^{19}$. Sin embargo, está por ver si crisneja se originó en Andalucía, lo cual sería posible de tratarse de la mera sufijación sobre una forma simple, pero no si es derivado directo de un lat. vg. ${ }^{*}$ crinicula, como Corominas supone; y, desde luego, dicha palabra bien pudo llegar al dominio andaluz con el habla de sus repobladores: es probable que algo indique en este sentido un crineja (o clineja) 'trenza de pelo' recogido por el mismo Alvar en el ciudadrealeño Viso del Marqués ${ }^{20}$. Todavía más, Pilar García Mouton y Francisco Moreno Fernández me facilitan los siguientes datos sobre la pervivencia del referido vocablo en Castilla la Nueva, como nombre de una tira de esparto trenzado en varios ramales:

Provincia de Cuenca: carneja (Arcos de la Sierra), cleineja (Belmontejo); provincia de Toledo: claineja (Camuñas, Villarrubia de Santiago), clarineja (Ocaña), clineja (Mora de Toledo, Yepes); provincia de Albacete: crineja (Alcaraz, Salobre), grisneja (Villaverde de Guadalimar); provincia de Ciudad Real: clineja (Alcolea de Calatrava, Villamanrique) ${ }^{21}$.

Esta colecta léxica resulta altamente ilustrativa desde el punto de vista histórico. En efecto, la dispersión de las variantes encuestadas y las profundas alteraciones fonéticas que presentan, algunas indudablemente debidas a cruces con otras voces, son hechos demostrativos de la gran populariza-

${ }^{19}$ Manuel Alvar, «Tradición lingüística andaluza en el Vocabulario de Nebrija», Boletín de la RSBAP, L-2, 1994, págs. 492, 497. Pero pleita se encuentra también al norte de Andalucía (v. n. 21), pudiendo tratarse de un andalucismo emigrado de su dominio originario, o de un mozarabismo común a buena parte del mediodía peninsular, La Mancha incluida.

${ }^{20}$ Manuel Alvar, El español hablado en Tenerife, Anejo LXIX de la RFE, 1959, pág. 159. Para la cuestión semántica no estará de más considerar el noroccidental enclines (también extremeño enclín 'crin') 'trampas hechas con redes para cazar perdices': Eugenio Miguélez Rodríguez, Diccionario de las hablas leonesas, León, 1993, pág. 278. Así mismo puede arrojar luz sobre la lógica de este proceso léxico-semántico el aragonés clin, no sólo 'crin', sino también 'fibra de esparto o de otras plantas con que se henchían colchones y jergones'. El ALEA en algunos puntos de Andalucía registra para la trenza de pelo los nombres de sogueta o soguilla.

${ }^{21}$ De los materiales que en encuestas de campo para el Atlas Lingüistico y Etnográfico de Castilla-La Mancha han recogido estos dos investigadores, a quienes agradezco su información. El número de ramales de la crisneja varía en las localidades encuestadas (entre 4 y 9 , según los datos de que dispongo), pero los tiene en menor número que la pleita donde este término se registra, concretamente en los pueblos toledanos de Camuñas y Villarrubia de Santiago. 
ción de este término, cuya antigua geografia castellano-nueva ha ido fragmentándose desde fechas por ahora indeterminadas. En consecuencia, seguramente no estamos frente a un término originariamente andaluz, sino irradiado más allá de Sierra Morena a partir del vecino dominio manchego. Bien sabido es, sin embargo, que muchos andalucismos lo son precisamente por haberse conservado en el mediodía peninsular mejor que en las zonas de las cuales los vocablos en cuestión procedían. Pero, de acuerdo con lo que sobre el particular sabemos, a los efectos de considerar el traslado de crisneja a Indias y su conversión en americanismo léxico, será conveniente pensar mejor en un meridionalismo como punto de partida, no sólo porque su mayor alcance dialectal se halla en consonancia con la realidad lingüística, sino porque a lo largo del siglo XVI pudieron ser muchos los castellanos nuevos emigrados al Nuevo Mundo que llevaron esta palabra en su bagaje idiomático. 